\title{
Comparing Defense Mechanisms, Resilience and Cognitive Distortion of Patients with Multiple Sclerosis and Healthy Individuals
}

\author{
Javad Setareh ${ }^{1}$, Mani Bahrami Monajemi ${ }^{2}$, Mahmood Abedini ${ }^{3}$, Masoumeh Tasha ${ }^{4} \&$ Sepehr Setareh $^{5}$ \\ ${ }^{1} \mathrm{MD}$, Assistant Professor of Psychiatry, Psychiatry and Behavioral Sciences Research Center, Addiction Institute, \\ Mazandaran University of Medical Sciences, Sari, Iran \\ ${ }^{2}$ MBPSs, Department of Clinical Psychology, University of Tehran, Tehran, Iran \\ ${ }^{3}$ MD, Department of Neurology, Clinical Research Development Unit of Bou Ali Sina Hospital, Mazandaran \\ University of Medical Sciences, Sari, Iran \\ ${ }^{4}$ M.A of Clinical Psychology, Mazandaran University of Medical Sciences, Sari, Iran \\ ${ }^{5}$ Medical Student, Shahid Beheshti University of Medical Sciences, Tehran, Iran \\ Correspondence: Mani Bahrami Monajemi (MBPSs), Department of Clinical Psychology, University of Tehran, \\ Tehran, Iran. E-mail: mani.b.monajemi@ut.ac.ir; mani.b.monajemi@warwickgrad.net
}

Received: June 13, 2017 Accepted: August 10, 2017 Online Published: August 21, 2017

doi:10.5539/gjhs.v9n10p44 URL: https://doi.org/10.5539/gjhs.v9n10p44

\begin{abstract}
Objective: The debilitating role of emotion and stress in multiple sclerosis (MS) has been highlighted in various literatures. The aim of this study is to evaluate role of defense mechanisms, resilience and cognitive distortion, which are considered influential factors with respect to stress management and emotion regulation among patients with MS.
\end{abstract}

Materials and Methods: This study consisted of 100 patients with MS and 100 healthy individuals in Sari (Mazandaran province, Iran), who were selected via convenient sampling method. In order to collect data, we used Defensive Style Questionnaire (DSQ40), Connor-Davidson Resilience Scale (CD_RISK) and Abdollahzadeh's cognitive distortions scale (CDS).

Results: According to results of currents study, there is no significant difference among people with MS and healthy individuals in term of using defense mechanisms at Mature and Neurotic levels (sig $>0.05)$ and there is significant difference between healthy people and people with MS with respect to immature level (sig $<0.05)$. The findings by Conner-Davidson Resilience Scale (CD-RISK) showed that the resilience of healthy people is more than people with MS (65/14 versus 57/5). The study also found that healthy people have more average cognitive distortion in comparing to MS patients (72/96 vs 67/7). Results showed that there is significant difference in overgeneralization, disqualifying the positives, emotional reasoning, expression of should and personalization among healthy people and people with multiple sclerosis $($ sig $<0.05)$ but there is no significant difference among other indicators of cognitive distortions (All or Nothing Thinking, Filtering, Jumping To Conclusion, Magnification versus Minimization and Labeling) ( $\mathrm{Sig}>0.05)$.

Conclusion: Current study, highlighted importance of evaluating psychological variables and their influences on quality of life among MS patients. It appears plausible that assessment of MS patients regarding their defense mechanisms, resiliency and cognitive distortion is essential; after aforementioned assessment clinicians can tailor suitable psychotherapeutic intervention with respect to exclusive needs of patients.

Keywords: defense mechanisms, resilience, cognitive distortion, multiple sclerosis

\section{Introduction}

MS is an autoimmune disease of the central nervous system (CNS) in which the immune system target its own tissue, particularly the nervous tissue (Philips \& Stuifbergen, 2008), consequential in damaged areas that are unable to transmit nerve impulses. The disease progressively damages areas responsible for the transmission of nerve impulses as well as the nerves themselves (The Columbia Encyclopedia, 2015).

The cause of this disease still remains baffling to neuroscientists and medical clinicians. The fundamental mechanism is thought to be either demolition by the immune system or failure of the myelin producing cells 
(Nakahara, Maeda, Aiso, \& Suzuki, 2012). MS is a disease that has been conceived as a result of viral, genetic, environmental, and psychological factors. A psychosocial factor (socioeconomic factor, occupation), which may predict the onset of multiple sclerosis, has an important role in the progression of the disease and its symptoms are closely entangled (Brad Shaw \& Rose, 2008).

A defense mechanism is a coping technique that decreases anxiety arising from unacceptable or potentially harmful impulses (Schacter, 2011), while the person is often not aware of these processes (Blaya, Dornelles, Blaya, Kipper, Heldet, Isolan, \& Band, 2006).

Andrews and Colleagues (1993) divided 20 defensive mechanisms into 3 central 'styles': developed, neurotic, and immature. The three styles are based on hierarchical classification. Immature defense mechanisms are demonstrative of inefficient and maladaptive coping methods (Andrews, Singh, \& Band, 1993).

Psychological resilience is described as a person's ability to properly adjust to stress and hardship (American Psychological Association, 2014). Resilience is commonly supposed as a "positive adaptation" after a stressful or traumatic situation (Hopf, 2010).

According to recent literatures, resilience is the result of individuals being able to interact with their environments and the processes that either promote well-being or protect them against the overwhelming influence of risk factors (Zautra, Hall, \& Murray, 2010). Patients with improved resilience were found to yield better treatment outcomes than patients with non-resilience focused treatment plans (Min, Yu, Lee, \& Chae, 2013). Cognitive distortions are thoughts that involuntarily appear in person, which maintain negative consequences in their emotion; also known as negative automatic thoughts (Dobson, 2010). Cognitive distortion is associated with postponing quick recovery in chronic diseases, which includes cognitive errors. In this sense, exaggerated generalization can be mentioned, which is highly correlated with physical and psychological disabilities. (Patrick \& Kerstin, 2011).

One of the main concerns of most patients with multiple sclerosis and their families is maintaining optimum life. The resilience of afflicted individuals depends on their perception of disease. Additionally, their cognitive distortions with respect to their disease, would lead to reduced quality of life, hope and a sense of resilience (Aghayousefi, Farhad, \& Mahdi, 2012).

As we explained earlier, defense mechanisms, resilience and cognitive distortion are factors, which can be predictive of psychological health and indirectly they may maintain an influence on physical health of patients as well. The results of this study can provide promising information for authorities and clinicians in order to promote quality of life among MS patients. Data resulted from our study, can facilitate multimodal approach toward this disorder.

\section{Materials and Methods}

This study is non-experimental ex post facto design. The population pool of this study consisted of 2100 individuals (both healthy and MS afflicted individuals) in Mazandaran Province. One hundred of patients with MS and 100 healthy individuals who had no history of chronic and severe disease were chosen via convenient sampling method, and they were paired in terms of gender and age. The samples of people aged 20 to 50 years of age and of both sexes were selected.

In order to collect the data needed and test the hypothesis we used proper measurement tools that were standard questionnaires. Data collection in this study made use of the Defense Style Questionnaire (DSQ-40), resilience questionnaire of Conner and Davidson (CD-RISK) and cognitive distortions questionnaire of Abdollahzadeh (CDS).

Defense Style Questionnaire (DSQ-40): A revised version of the original scale was developed in 1993 by Andrews and colleagues. The questionnaire consisted of 40 questions and evaluates 20 defense mechanisms at three levels: mature, neurotic and immature. The validity of Defensive style questionnaire was confirmed by reliability method and using Cronbach's alpha (Andrews et al., 1993; Sinha \& Watson, 2004).

Resilience questionnaire by Conner-Davidson (CD-RISK): was made to delineate various dimensions of resilience and regarding clinical implications. Resilience is the power to deal with adverse circumstances. The CD-RISC is a 25 item scale that has been used in a various of contexts such as, members of different ethnic groups and cultures, neurologic patients caregivers, adolescents, elders, patients in treatment for PTSD, military medical personnel, medical students, college students, survivors of various traumas, social workers, and even select professional or athletic groups. Though the means scores tends to vary with respect to different settings and environment, the psychometric characteristics of this scale, tend to be applicable to almost any settings. There are two more brief versions of the 25 -item Connor-Davidson Resilience Scale, there is a 10-item (CD-RISC 10). The 
CD-RISC 10 can range in score from $0-40$ and is made of questions $1,4,6,7,8,11,14,16,17$, and 19 from the original 25 -item scale. In a study conducted by Samani et al (1386), the reliability of this scale was estimated $87 \%$ via using Cronbach's alpha coefficient (Samani, Jokar, \& Sahragard, 2007). Furthermore, Mohammadi (1384) estimated coefficient reliability as $89 \%$ via using Cronbach's alpha and obtained validity via estimating correlation of all part of the scale with total score between $41 \%$ to $64 \%$ and they concluded that this test maintain suitable internal consistency in Iran population (Mohammadi, Rahimi, \& Mohammadi, 2011).

Cognitive distortions questionnaire of Abdollahzadeh and Salar (CDS): This questionnaire is called cognitive distortions questionnaire. This device provides awareness of cognitive distortion in daily life. Most of our problems and inappropriate feelings are due to irrational thinking. Identifying these thinking patterns is necessary in order to eliminate these interfering thoughts. The questionnaire is consisted of 20 words introduced by Albert Ellis that measure cognitive distortions and any irrational thinking allocated 2 expressions The Cognitive Distortions Questionnaire showed satisfactory internal consistency (Cronbach's alpha $=0.85$ ) in Iran population (Abdullah \& Salar, 2009).

\section{Findings}

According to data in Table 1, via using Defensive Style Questionnaire (DSQ-40) it can be inferred that there is no significant difference among people with MS and healthy individuals in term of using defense mechanisms at Mature and Neurotic levels (sig $>0.05$ ) and there is significant difference between healthy people and people with MS with respect to immature level (sig<0.05).

Table-1. Summary Results of T-Test in two independent groups in comparing parameters of defense mechanisms among Healthy people and patients with MS

\begin{tabular}{llllllll}
\hline Variable & Groups & Number & Mean & SD & T & DOF & Meaningfulness \\
\hline Mature & Healthy & 100 & 112.84 & 24.02 & -1.947 & 198 & 0.053 \\
& MS & 100 & 119.76 & 26.18 & & & \\
Neurotic & Healthy & 100 & 42.94 & 9.345 & 0.570 & 198 & 0.570 \\
& MS & 100 & 42.10 & 11.41 & & & \\
Immature & Healthy & 100 & 41.40 & 9.46 & $-2.306^{*}$ & 198 & 0.022 \\
& MS & 100 & 44.88 & 11.75 & & & \\
\hline
\end{tabular}

According to Table 2, Conner-Davidson Resilience Scale (CD-RISK) showed that the resilience of healthy people is more than people with MS (65/14 versus 57/5). The value difference is considered meaningful by T-test because the significance level $(\mathrm{Sig}=0 / 001)$ error was smaller than $\% 05$. Hence, we can say with $95 \%$ confidence there is a significant difference between the resilience in people with MS and healthy individuals.

Table 2. Summary of T-test results for comparing resilience of healthy people and people with MS

\begin{tabular}{llllllll}
\hline Variable & Categories & Number & Mean & SD & T & DOF & Level \\
\hline Resiliency & Healthy & 100 & 65.14 & 12.97 & $3.453^{* *}$ & 198 & $\mathbf{0 . 0 0 1}$ \\
& MS & 100 & 57.50 & 17.92 & & & \\
\hline
\end{tabular}

According to Table 3 , it appears plausible that healthy people have more average cognitive distortion in comparing to MS patients (72/96 versus 67/7). The value difference T-test is significant because the significance level ( $\mathrm{Sig}=$ $0 / 003$ ) was smaller than $\% 05$ errors with 95 percent confidence, so the null hypothesis has been rejected and the research hypothesis is confirmed. In other words there is a significant difference between the cognitive distortion among people with MS and healthy individuals. Results showed that there is significant difference in overgeneralization, disqualifying the positives, emotional reasoning, expression of should and personalization among healthy people and people with multiple sclerosis (sig $<0.05)$ but there is no significant difference among other indicators of cognitive distortions (All or Nothing Thinking, Filtering, Jumping To Conclusion, Magnification versus Minimization and Labeling) ( $\mathrm{Sig}>0.05$ ). 
Table 3. Summary of T-test results in two independent groups, comparing indicators of cognitive errors between healthy people and people with MS

\begin{tabular}{llllllll}
\hline Variable & Groups & Number & Mean & SD & T & DOF & Meaningfulness \\
\hline All or Nothing Thinking (Splitting) & Healthy & 100 & 7.82 & 1.43 & 0.959 & 198 & 0.339 \\
& MS & 100 & 7.62 & 1.516 & & & \\
Exaggerated Generalization & Healthy & 100 & 7.40 & 2.23 & $2.175^{*}$ & 198 & 0.031 \\
& MS & 100 & 6.74 & 2.06 & & & \\
Filtering & Healthy & 100 & 6.70 & 1.99 & 0.790 & 198 & 0.430 \\
& MS & 100 & 6.50 & 1.56 & & & \\
Disqualifying the Positives & Healthy & 100 & 8.18 & 1.38 & $3.684^{* *}$ & 198 & 0.000 \\
& MS & 100 & 7.28 & 2.01 & & & \\
Jumping To Conclusion & Healthy & 100 & 6.96 & 1.85 & 1.587 & 198 & 0.114 \\
ES & MS & 100 & 6.52 & 2.06 & & & \\
Healthy & 100 & 6.98 & 2.12 & $2.631^{* *}$ & 198 & 0.009 \\
Magnification & MS & 100 & 6.26 & 1.73 & & & \\
versus & Healthy & 100 & 7.70 & 1.96 & 1.601 & 198 & 0.111 \\
Minimization & MS & 100 & 7.28 & 1.74 & & & \\
Should Statement & Healthy & 100 & 5.94 & 1.75 & $2.742^{* *}$ & 198 & 0.007 \\
Labeling & MS & 100 & 5.22 & 1.95 & & & \\
Personalization & Healthy & 100 & 7.84 & 1.91 & 1.067 & 198 & 0.287 \\
& MS & 100 & 7.56 & 1.79 & & & \\
\hline & Healthy & 100 & 7.44 & 1.49 & $3.543^{* *}$ & 198 & 0.000 \\
& MS & 100 & 6.72 & 1.37 & & & \\
& & & & & & &
\end{tabular}

\section{Discussion}

The relationship between psychological discomforts and MS symptoms has been undervalued in the past, but there is growing evidence of elevated interest within the scientific community. For instance, various literature is suggesting that psychological stress boosts relapse risk in MS (25). We tend to believe that more comprehensive and thorough investigations into the physiological outcomes of a psychological intervention may yield a better understanding of therapeutic options for people with MS.

In a study conducted by Pagnini and Colleagues, they assessed role of psychological intervention among MS patients on both physical and mental symptoms. In their study they concluded that improving psychological factors such as defense mechanism, cognitive distortion and resilience among MS patients will not only influence psychological state of this states and would reduce anxiety and depression; but psychological interventions would also maintain positive impacts on physical symptoms such as fatigue and pain. In their study they concluded that MS patients tend to show lower resilience comparing to healthy individuals (Francesco, Colin, Deborah, \& Ellen, 2014). It is still unclear, whether decreased resilience stems from disease itself or it can be referred to socio-economic factors, age, gender or other possible variables.

Results of this study showed that the average defense mechanisms in patients with multiple sclerosis are more than healthy people (206/74 to 197/18). The independent t-test showed this value difference.

[Everyone use defense mechanisms in order to defeat anxiety, compensate their deficiencies and reduce their failure rate. Defense mechanisms ranges from mature to pathological styles and different individuals tend to use various mechanisms in order to cope with stress and anxiety (Vaillant, 2000).

Resiliency average of healthy people is more than people with multiple sclerosis $(65 / 14$ versus $75 / 5)$. The $t$ independent test confirmed this result/. In other words there is a significant difference between the resilience in people with MS and healthy individuals and patients with multiple sclerosis has lower resilience compared to 
healthy cohort. The findings of other studies have shown that there is a significant correlation between resiliency and vulnerability in patients with multiple sclerosis (Devika, Amanda, \& Taylor, 2016) and resilience can predict the quality of life of patients with multiple sclerosis (Michaeli, Mokhtarpourhabashi, \& Meysami, 2013).

Harrell and Achiron (2014) studied patients with multiple sclerosis cognitive resilience relapsing - remitting; they concluded that cognitive resilience in people with multiple sclerosis relapsing - remitting is low. The results showed that people who have lower resiliency tend to experience more attacks and the people with higher level of resiliency show more promising recovery (Harel \& Achiron, 2009).

Posse and Knopf (2011), in a study titled Effort for integrating the three cognitive model of depression' find the conclusion that cognitive errors contribute in the absence of rapid healing of chronic diseases. Including effective cognitive distortion can be named overgeneralization highly correlated with physical and psychological disabilities. T-test results showed that there is no significant difference between people with MS and healthy individuals in the use of defense mechanisms at the level of neuroticism and mature. But there is a significant difference in the use of immature defense mechanisms among people with MS and healthy individuals $(\mathrm{Sig}<0.05 \%)$. In other words, people with multiple sclerosis use a greater proportion of immature defense mechanisms (Patrick \& Kerstin, 2011)

According to one survey, mentally healthy people use more developed defense mechanisms and people with many negative indicators such as personality disorder and depression tend to use immature defense mechanisms (Vaillant, 2000).

The t-test results in this study showed that there is significant difference between patients with MS and healthy individuals in the use of cognitive distortion, significant differences in indicators overgeneralization, discounting positive, emotional reasoning, should and better expressions and personalization ( $\mathrm{Sig}>0.05 \%)$. But there is no significant difference in other indicators of cognitive distortions such as all-or-nothing thinking, negative filter, hasty conclusions, macroscopic-microscopic and labeling ( $\mathrm{Sig}>0.05 \%$ ).

Ebrahimi, Zarinedini and Meracy (2010), in a study named relationship between psychopathology family ties with cognitive vulnerability (dysfunctional attitudes and interpersonal cognitive distortions) concluded that there is significant difference between the cognitive distortions the individual, irrational beliefs in marriage and dysfunctional attitudes relationship with marital conflicts. A marital conflict was predictable based on scores on cognitive distortions, irrational beliefs on marriage and dysfunctional attitudes (Fischer, Heesen, \& Gold, 2011).

Finally, in a study conducted by Bahrami Monajemi and Colleagues (2015) they studied various factors such as personality traits, mood disturbance and disease severity and defense mechanisms. The result of their study is consistent with the result of current study. MS patients tend to have lower resiliency and more problematic cognitive processing (Francesco et al., 2014).

The findings of this study showed in patients with multiple sclerosis have lower resiliency than healthy people have more resilience. Also there is a significant difference among people with MS and healthy people in the use of cognitive distortions. The findings showed that people with multiple sclerosis use a greater extent of overgeneralization of cognitive distortions, discounting positives, emotional reasoning, should and better expressions and personalize than healthy people.

\section{Conclusion}

Current study, highlighted importance of evaluating psychological casual roles on quality of life among MS patients. It appears plausible that assessment of MS patients regarding their defense mechanisms, resiliency and cognitive distortion is inevitable; after aforementioned assessment clinicians can tailor assuring psychotherapeutic intervention with respect to specific needs of the patients.

\section{Recommendations}

It is advisable to assess causal role of psychological dimensions with respect to other neurological disorder such chronic pain, fibromyalgia and Parkinson.

\section{Limitations of Study}

It would have been better to conduct current study among larger statistical sample.

\section{Authors' contributions}

JS and MBM conceived and designed the evaluation and they conducted whole process of this study. MA and MS collected and interpreted the clinical data and drafted the manuscript. SS Participated in conducting statistical evaluation. All authors read and approved the final manuscript. This paper was derived and inspired from master 
thesis dissertation number: 20820701932088

\section{Acknowledgements}

We would like to thank all patients who participated in the study.

\section{Competing Interests Statement}

The authors declare that there are no competing or potential conflicts of interest.

\section{References}

Abdullah, Z. H., \& Salar, M. (2009). Construction and validation of a questionnaire cognitive distortions. Tehran: The Institute of testYare pooya.

Aghayousefi, A. S., Farhad, D., \& Mahdi, B. Z. (2012). Its asset quality of life and psychological perception of disease among patients with MS. Journal of Health Psychology, 1, 1-15.

American Psychological Association. (2014). The Road to Resilience.

Andrews, G., Singh, M., \& Band, M. T. (1993). The defense style questionnaire. Journal of nervous and mental disease, 246-256. https://doi.org/10.1097/00005053-199304000-00006

Blaya, C., Dornelles, M., Blaya, R., Kipper, L., Heldet, E., Isolan, L., \& Band, M. (2006). Do defense mechanisms vary according to the psychiatric disorder. Rev Braspsiquiatr, 28(3), 179-183. https://doi.org/10.1590/S1516-44462006000300007

Brad Shaw, J., \& Rose, A. (2008). Cognition, Depression and Fatigue Multiple Sclerosis. ACNR, 8(4), 15-17.

Conner, K. M., \& Davidson, J. R. T. (2003). Development of a new resilience scale: The Conner - Davidson Resilience Scale (CD - RISC). Depression and Anxiety, 18, 76-82. https://doi.org/10.1002/da.10113

Devika, D., Amanda, S.-Z., \& Taylor, L. (2016). The Impact of Hope and Resilience on Multiple Factors in Neurosurgical Patients. Retrieved July, 2017 from https://www.ncbi.nlm.nih.gov/pmc/articles/PMC5120968/

Dobson, K. S. (2010). Handbook of Cognitive - behavioral Therapies. New York, London: The Guilford Press.

Ebrahimi, A., Sheikhzarinedini S., \& Meracy, M. (2012). Its psychopathology family ties with cognitive vulnerability (dysfunctional attitudes and interpersonal cognitive distortions). Journal of Isfahan, 29(167), $1-9$.

Fischer, A., Heesen, C., \& Gold, S. M. (2011). Biological outcome measurements for behavioral interventions in multiple sclerosis. Ther Adv Neurol Disord, 4, 217-229. https://doi.org/10.1177/1756285611405252

Francesco, P., Colin, M. B., Deborah, P., \& Ellen, L. (2014). Symptom changes in multiple sclerosis following psychological interventions: a systematic review. Retrieved November, 2016 from https://www.ncbi.nlm.nih.gov/pmc/articles/PMC4253984/

Harel, Y., \& Achiron, A. (2009). Cognitive resiliency in relapsing - remitting multiple sclerosis patients. Journal of the Neurological Sciences, 285, 1, 212-228. https://doi.org/10.1016/S0022-510X(09)70811-1

Hopf, S. M. (2010). Risk and Resilience in Children Coping with Parental Divorce. Dartmouth Undergraduate Journal of Science.

Michaeli, N., Mokhtarpourhabashi, E., \& Meysami, S. (2013). The role of social support, coping strategies and resilience in anticipation of the quality of life of patients with multiple sclerosis. New findings in the Journal of Psychology, 7(23), 5-17.

Min, J. A.; Yu, J. J.; Lee, C. U., \& Chae, J. H. (2013). Cognitive emotion regulation strategies contributing to resilience in patients with depression and/or anxiety disorders. Comprehensive Psychiatry, 54(8). https://doi.org/10.1016/j.comppsych.2013.05.008

Mohammadi, Rahimi, \& Mohammadi. (2011). Factors affecting the resiliency in individuals exposed to trauma. clinical psychology research and consulting, 1(61),5-14.

Nakahara, J., Maeda, M., Aiso, S., \& Suzuki, N. (February 2012). Current concepts in multiple sclerosis: autoimmunity versus oligodendrogliopathy. Clinical reviews in allergy \& immunology, 42(1), 26-34. https://doi.org/10.1007/s12016-011-8287-6

Patrick, P., \& Kerstin, K. (2011). Bridging the gaps: An attempt to integrate three major cognitive depression models. $\quad$ Retrieved July, 2017 from http://ir.library.louisville.edu/cgi/viewcontent.cgi?article=1156\&context=faculty 
Philips, L. J., \& Stuifbergen, A. (2008). The influence of positive Experiences on Depreession Volume. Nursing Hollstic of jurnal. MS with persons in life of quality and 26 Number, 1, 41.

Samani, S., Jokar, B., \& Sahragard, N. (2007). Resilience, mental health and life satisfaction. Iranian Journal of Psychiatry and Clinical Psychology, 50, 251-262.

Schacter, D. L. (2011). Psychology (2nd, ed., pp. 482-483). 41 Madison Avenue, New York, NY 10010: Worth Publishers.

Sinha, B. K., \& Watson, D. C. (2004). Personality disorder clusters and the Defense Style Questionnaire. Psychology and Psychotherapy, 77, 55-66. https://doi.org/10.1348/147608304322874254

The Columbia Encyclopedia (6th ed.). Copyright $\odot$ 2015, The Columbia University Press.

Vaillant, G. E. (2000). Adaptive mental mechanisms: Their role in a Positive Psychology. American Psychologist, 55, 89-98. https://doi.org/10.1037/0003-066X.55.1.89

Zautra, A. J., Hall, J. S. \& Murray, K. E. (2010). Resilience: A new definition of health for people and communities", pp. 3-34 in J.W. Reich, A. J. Zautra, \& J. S. Hall (eds.), Handbook of adult resilience. New York: Guilford.

\section{Copyrights}

Copyright for this article is retained by the author(s), with first publication rights granted to the journal.

This is an open-access article distributed under the terms and conditions of the Creative Commons Attribution license (http://creativecommons.org/licenses/by/4.0/). 